Published in final edited form as:

J Pain. 2013 December ; 14(12 0): . doi:10.1016/j.jpain.2013.06.001.

\title{
General health status and incidence of first-onset temporomandibular disorder: OPPERA prospective cohort study
}

\author{
Anne E. Sanders, \\ University of North Carolina at Chapel Hill, Chapel Hill, North Carolina \\ Gary D. Slade, \\ University of North Carolina at Chapel Hill, Chapel Hill, North Carolina \\ Eric Bair, \\ University of North Carolina at Chapel Hill, Chapel Hill, North Carolina
}

Roger B. Fillingim,

University of Florida, College of Dentistry, Gainesville, Florida

Charles Knott,

Battelle Memorial Institute, Center for Analytics and Public Health, Durham, North Carolina

Ronald Dubner,

University of Maryland Dental School, Baltimore, Maryland

Joel D. Greenspan,

Departments of Oral-Maxillofacial Surgery and Neural and Pain Sciences, and Brotman Facial

Pain Center, University of Maryland Dental School, Baltimore, Maryland

William Maixner, and

University of North Carolina at Chapel Hill, Chapel Hill, North Carolina

Richard Ohrbach

Department of Oral Diagnostic Sciences, University at Buffalo, State University of New York, Buffalo, New York

\section{Abstract}

Temporomandibular disorders (TMD) overlap with other health conditions but no study has examined which of these conditions increase the risk of developing first-onset TMD. The authors prospectively evaluated the relationship between health status at enrollment and subsequent incidence of TMD in 2,722 men and women. Participants aged 18-44 years had no history of TMD and were clinically free of TMD when enrolled in 2006-08 at four U.S. study sites in the

(C) 2013 The American Pain Society. Published by Elsevier Inc. All rights reserved.

Author for correspondence: Anne E. Sanders MS, PhD, MS, Department of Dental Ecology, School of Dentistry, Room 4502 Koury Oral Health Sciences Building, CB \#7450 Chapel Hill, NC 27599-7450. Telephone: [Int +1] 919-537-3275.

anne_sanders@dentistry.unc.edu.

\section{DISCLOSURES}

This work was supported by NIH grant U01DE017018 and P01NS045685. The OPPERA program also acknowledges resources specifically provided for this project by the respective host universities: University at Buffalo, University of Florida, University of Maryland-Baltimore, and University of North Carolina-Chapel Hill. Roger Fillingim and Gary Slade are consultants and equity stock holders, and William Maixner and Dr. Luda Diatchenko are cofounders and equity stock holders in Algynomics, Inc., a company providing research services in personalized pain medication and diagnostics. Other authors declare no competing interests.

Publisher's Disclaimer: This is a PDF file of an unedited manuscript that has been accepted for publication. As a service to our customers we are providing this early version of the manuscript. The manuscript will undergo copyediting, typesetting, and review of the resulting proof before it is published in its final citable form. Please note that during the production process errorsmaybe discovered which could affect the content, and all legal disclaimers that apply to the journal pertain. 
OPPERA prospective cohort study. First-onset examiner-classified TMD developed in 260 people over a median 2.8 years of follow-up. Cox regression estimated the association between health conditions and TMD incidence while accounting for potential confounders. Incidence of firstonset TMD was 50\% higher for people with lower back pain (adjusted hazard ratio [AHR] $=1.50$, 95\% confidence limits $[95 \% \mathrm{CL}]: 1.08,2.10)$ and $75 \%$ higher for people with genital pain symptoms (AHR $=1.75$ [95\% CL: 1.04, 2.93]) than people without a history of these pain disorders. Digit ratio, a marker of intra-uterine exposure to sex hormones, was significantly associated with TMD incidence. Other independent predictors of first-onset TMD were sleep disturbance and cigarette smoking. These findings reveal multiple-influences of health status on incidence of first-onset TMD.

\section{Keywords}

Temporomandibular Joint Disorders; Prospective Cohort Studies; Comorbidity; Epidemiology

\section{INTRODUCTION}

The observation, made 30 years ago, that temporomandibular disorders (TMD) coexist with other health conditions in some individuals ${ }^{31}$ challenged the assumption that these conditions were clinically distinct phenotypes. Today evidence from clinical and epidemiologic studies shows that TMD coexists with numerous regional and systemic disorders including fibromyalgia, ${ }^{1,25}$ low back pain/spinal pain, ${ }^{33,}, 47$ vulvar lichen sclerosus, ${ }^{5}$ chronic fatigue syndrome, ${ }^{11}$ irritable bowel syndrome, ${ }^{2}$ sleep disorders, ${ }^{44}$ nonulcerative interstitial cystitis/painful bladder syndrome, ${ }^{37}$ tension-type and migraine headaches, $,{ }^{9}, 19,20$ allergy conditions, ${ }^{41}$ and somatoform disorders. ${ }^{17}$ What is striking about this evidence is not so much the diversity of these conditions that span autonomic, atopic and sensory disorders, but rather the homogeneity among them that is defined by three clinical features. Firstly most of these disorders share an idiopathic basis in which the pathophysiologic mechanisms are poorly understood. ${ }^{12,27}$ Secondly, the clinical presentation of most of these disorders exhibits a disproportionate level of symptom severity, including pain and fatigue, compared to examination findings. Thirdly, most share demographic, social, behavioral and psychological features. The presence of shared features, including possibly etiology as well, in these joint relationships has prompted use of the term "overlapping" conditions and many studies have quantified the extent of this overlap. $1,210,34,39,42$

Evidence of pain disorders overlapping with TMD was demonstrated in the Orofacial Pain Prospective Evaluation and Risk Assessment (OPPERA) baseline case control study of chronic TMD in adults aged 18-44 years. ${ }^{35}$ There, 1633 asymptomatic controls, examined using Research Diagnostic Criteria for TMD ${ }^{14}$ (RDC/TMD) and found not to have TMD, were compared with 185 adults meeting RDC/TMD criteria for chronic painful TMD. In chronic TMD cases compared to controls, odds of irritable bowel syndrome were 2.7 times as high (95\% confidence limits [CL]: 1.4, 5.1), odds of chronic pain in the body other than the face were 5.1 times as high (95\% CL: 3.6, 7.3), and odds of any headache in the last year were 8.8 times as high $(95 \%$ CL: $3.8,20.1)$ after statistically adjusting for the potential confounding effects of age, gender and race/ethnicity. ${ }^{35}$ Moreover some effects were dosedependent. For example, using an ordinal count of different types of headache experienced in the previous year, adjusted odds of one type of headache relative to no type of headache were 3.9 times as high $(95 \%$ CL: 1.6, 9.7) for TMD cases compared to controls. Odds of two headache types relative to none were 7.8 times as high $(95 \%$ CL: 3.3, 18.6) for TMD cases, and odds of three headache types relative to none were 15.0 times as high (95\% CL: 6.1, 36.6) in TMD cases as for controls. ${ }^{35}$ 
On one level this constellation of relationships poses challenges for the identification of pathophysiologic mechanisms and the development of multimodal treatment strategies. Another challenge is to distinguish cause and effect: which clinical conditions are preexisting experiences that contribute to development of TMD and which, such as sleep disorders or mood disorders, may be consequences of the disorder.

Until now longitudinal studies have not established which overlapping conditions predispose an individual to greater risk of first-onset TMD. To address that question, this paper reports findings from the OPPERA prospective cohort study. The aim of this analysis was to determine prospectively which clinical conditions observed at baseline in TMD-free individuals were associated with higher risk of developing first-onset TMD.

\section{MATERIALS AND METHODS}

The OPPERA study is a prospective cohort study designed to investigate the etiology of first-onset TMD. The OPPERA cohort comprised 3,263 adults with no history of TMD were enrolled at four US study sites and followed for a median 2.8 years, during which time 260 of them developed first-onset, examiner-classified TMD.

Institutional review boards at each study site granted approval for study procedures and signed, informed consent was obtained from each subject. Full details of enrollment, followup and statistical analyses are provided elsewhere in this volume ${ }^{4}$ and are summarized here.

\section{Recruitment, Eligibility Criteria and Enrollment}

Between May 2006 and November 2008, potential study participants were recruited using advertisements, emails and flyers at four U.S. study sites: Baltimore, MD; Buffalo, NY; Chapel Hill, NC; and Gainesville, FL. Eligibility criteria were age 18-44 years, good health, no history of facial injury or surgery, no significant symptoms of TMD pain, no previous diagnosis of TMD, and an absence of TMD myalgia and TMD arthralgia on clinical examination. On enrollment participants completed a telephone interview and selfadministered questionnaires assessing hypothesized risk factors for TMD. During a threehour clinical visit, autonomic function was monitored and quantitative sensory tests measured sensitivity to painful stimuli. Study examiners recorded clinical characteristics of muscles and joints of the head, neck and body and they verified absence of TMD.

\section{Scope of this Analysis}

This analysis examined clinical conditions that may be potential putative risk predictors of first-onset TMD. Since very little is known about clinical predictors, we included a wide range of baseline clinical characteristics summarized as pain disorders, clinical status indicators, sleep quality and anthropometric indicators. These were selected on a priori basis of theoretical relevance or evidence of their association with TMD. Their measurement is described below. Conditions involving face or jaw symptoms are reported elsewhere in this volume (see Ohrbach et. al.). Further information about the measures and forms used in OPPERA are available on-line at the Journal of Pain as part of the supplement to the Ohrbach et al, 2011 publication. ${ }^{35}$ See URL: http://download.journals.elsevierhealth.com/ mmcs/journals/1526-5900/PIIS1526590011007437.mmc1.pdf

\section{Pain Disorders}

Symptoms of pain disorders were evaluated using batteries of items in the OPPERA Comprehensive Pain and Symptom Questionnaire (CPSQ) published in OPPERA baseline supplementary material. ${ }^{35}$ This analysis draws on pain symptoms related to headache, lower back pain, irritable bowel syndrome and genital symptoms. Headache was classified 
according to the second edition of the International Classification of Headache Diseases (2004) developed by the International Headache Society. We examined three types of primary headache: probable tension-type, tension-type, and migraine headache; while the headache symptoms (OPPERA CPSQ questions 37 through 41) were classified algorithmically according to ICHD-2 decision-rules into the various subtypes (e.g., episodic tension-type (TT), chronic TT, probable migraine), only the main types listed here were considered for the present analyses. Past or current lower back pain symptoms were assessed in the OPPERA CPSQ question 51. These items evaluated the constancy of pain symptoms, the frequency of episodes in the last 12 months, the duration of episodes, and whether pain symptoms extended into the buttocks or legs. Assessment of irritable bowel syndrome (IBS) symptoms was based on Rome III criteria in CPSQ questions 52 and 53. The IBS questions asked about bowel movements and the experience of discomfort or pain in the abdomen that lasted at least one day a week during the previous three weeks. Genital symptoms were assessed with CPSQ questions 54 through 57 that asked about the presence of genital pain on contact but absence of genital itching during the last three months CPSQ question 54.

\section{Health Status}

A subjective assessment of health status obtained in the medical history questionnaire asked people to describe their health overall as excellent, good, fair or poor. A checklist of medical conditions asked respondents to indicate conditions they had now or in the past. Conditions were grouped as: (1) endocrine conditions (diabetes; hypothyroid disease; hyperthyroid disease); (2) cardiovascular conditions (mitral valve prolapse; high blood pressure; angina; heart attack; heart failure; pacemaker/defibrillator; stroke); (3) hematologic conditions (anemia; bleeding disorder; leukemia); (4) neural and sensory conditions (earache, ringing in ears; hearing loss; fainting or dizzy spells; epilepsy, seizures, or convulsions; psychiatric treatment); (5) respiratory conditions (sinus trouble; allergies or hives; asthma; tuberculosis; breathing difficulties.) Finally a binary response (yes/no) was used for each of sleep apnea, and history of being hospitalized for any surgical operation or serious illness. A count of 11 currently used medications was computed.

\section{Pittsburgh Sleep Quality Index (PSQI)}

The PSQI ${ }^{7}$ investigated whether disturbances in sleep play a role in development of firstonset TMD. Participants rated their sleep quality and disturbances over a one-month reference period. Scores for the seven PSQI components- subjective sleep quality, sleep latency, sleep duration, habitual sleep efficiency, sleep disturbances, use of sleeping medication, and daytime dysfunction-were summed to yield one global score. Sleep disorders are associated with headache ${ }^{40}$ and primary insomnia is associated with TMD. ${ }^{44}$

\section{Anthropometric Status}

In the physical examination weight and height were measured using standardized equipment to compute body mass index $\left(\mathrm{BMI}=\right.$ weight $/$ height $\left.^{2}\right)$. In a U.S. population health survey, a strong dose-response positive relationship was observed between BMI and pain experienced during the previous day for "a lot of the day". 45

Examiners used photocopied images of participants' hands to measure the length of the second digit [2D] and the fourth digit [4D]). The 2D:4D ratio was calculated and averaged for both hands. A high 2D:4D ratio is a marker of greater estrogen relative to testosterone exposure toward the end of the first trimester in utero. ${ }^{28}$ 


\section{Cigarette Smoking}

People who reported having smoked fewer than 100 cigarettes in their lives were classified as non-smokers, and the remainder were asked whether they were current or former smokers. In the Kentucky Women's Health Registry, daily smoking showed a dose-response relationship with at least one pain syndromes among fibromyalgia, sciatica, chronic neck pain, chronic back pain, joint pain, chronic head pain, nerve problems, and pain all over the body. ${ }^{30}$ In that study, former smoking showed a weaker effect than daily smoking, but remained a significant risk factor. Previously we reported a strong association between cigarette smoking and chronic TMD. ${ }^{41}$

\section{Short Form 12 Health Survey v2 (SF-12v2)}

General health status was evaluated using the Short Form 12 Health Survey v2 (SF-12v2). This analysis used the weighted summary scores that comprise the physical component (PCS-12) and the mental component (MCS-12) derived using the standard SF-12V2 scoring algorithm. Like self-rated health, this measure was excluded from multivariable models since it is considered to be a consequence of health conditions, rather than an independent determinant.

\section{Follow-up and Case-Classification of First-Onset TMD}

At three-monthly intervals after enrollment through May 2011, study participants were asked to complete a questionnaire that screened for TMD pain symptoms. Those who reported TMD pain symptoms were asked to attend a clinical examination that determined presence or absence of painful TMD according to OPPERA's adaptation of the RDC/TMD criteria. ${ }^{14}$ In this adaptation, classification of first-onset TMD required two criteria: (1) $\geq 5$ days/month of pain in TMD locations specified by examiner; and (2) examiner findings of arthralgia (i.e., pain in temporomandibular joint(s) during jaw maneuver or digital palpation) or myalgia (i.e., pain during jaw maneuver or digital palpation in 23 of 8 muscle groups, each assessed bilaterally: temporalis, masseter, lateral pterygoid, submandibular) or both.

All examiners underwent annual training and calibration in the RDC/TMD protocol. In blinded, replicated examinations, Kappa statistics for inter-examiner reliability of TMD case-classification ranged from 0.87 to 1.0 , signifying excellent reliability.

\section{Statistical Analysis}

All analyses were conducted with SAS version 9 statistical software (SAS Institute, Inc, Cary, North Carolina). The followup period for each study participant was computed as the time from enrollment to the first of three possible events: 1) examiner-classification of firstonset TMD; 2) loss to follow-up; or 3) the census date used for this analysis (i.e., May, 2011). Incidence of first-onset TMD was calculated as the number of people with first-onset TMD divided by the sum of follow-up periods, and the result was expressed as the percent of people per annum (equivalent to the number of incident cases per 100 years of followup). For descriptive purposes, an adjusted, average annual incidence of firstonset TMD was computed using a Poisson regression model that adjusted for study site. Buffalo was selected as the referent from among the four study sites.

To test hypotheses about associations between baseline risk factors and TMD incidence, hazard ratios were computed as approximations of the rate ratio. Hazard ratios represent the relative difference in hazard rates between two groups. While the hazard rate is a theoretical construct, representing the instantaneous probability of an event as the duration of follow-up approaches zero, it is a good approximation of the average rate ratio in a cohort study. Furthermore, the Cox proportional hazards models used to estimate hazard ratios require fewer statistical assumptions than other modeling methods. Hereafter, we use the term 
"incidence" when referring to the annual incidence rate and the hazard rate. We report Pvalues unadjusted for multiple tests. However a strict Bonferroni correction that took account of the 26 variables reported in univariate results (Tables 1,2 and 3), would revise the critical $\mathrm{P}$-value to $\mathrm{P}<0.002$.

For the Cox models, incident cases of TMD were regarded as an event; otherwise they were censored. Each person's follow-up period was used as the time-to-event. When the baseline risk factor was categorical, one category was nominated as the referent and dummy variables represented each of the other categories. For continuous variables, scores were transformed to unit-normal deviates (mean $=0$, standard deviation=1). This transformation to standardized values permits a comparison of the magnitudes of effect of the risk factors when these are measured on different continuous scales, although not when measured categorically. When calculating univariate hazard ratios and $95 \%$ confidence limits, a hierarchical approach was used in which models initially adjusted only for study site, and subsequently additionally adjusted for demographic characteristics, i.e., age in years, gender, race/ethnicity (white, African American/Black, Hispanic, Asian, and other/unstated), and lifetime U.S. residence (no, and yes/unstated).

Hazard ratios were also computed using multiple imputation to account for two sources of potential bias associated with: a) non-examination of 243 people with symptoms; and b) a higher-than expected incidence of TMD classification for one examiner who conducted 75 examinations. As described in detail elsewhere, ${ }^{4}$ findings for 318 examinations were imputed in three steps. First, an algorithm predicting probability of TMD was created using binary logistic regression analysis of quarterly screening questionnaire data among people who were re-examined. Predictor variables in the algorithm were: study site, time since enrollment, and number of non-specific orofacial symptoms reported in the quarterly health update. The algorithm was then used to generate 100 imputed, binary case-classifications for each person who was not examined, or whose examination findings were discarded. Finally, the imputed case classifications and 100 replicates of observed case-classifications were analyzed in a Cox regression model using multiple imputation to estimate an average hazard ratio and corresponding $95 \%$ confidence interval. The imputed analysis adjusted for OPPERA study and demographic characteristics described above. When fitting the random forest models, the 318 people used for imputation were given missing censoring indicators and imputed using adaptive tree imputation.

Variables found to be significantly associated with TMD onset in the demographicallyadjusted, imputed analysis were evaluated for their combined effects in successive multivariable models. The first model considered only the socio-demographic characteristics; the second model added painful conditions, and the third model added nonpainful conditions. All variables were retained in the model, regardless of their statistical significance. Instead, the focus was on the degree to which estimated hazard ratios were altered by addition of new blocks of variables, signifying likely confounding. Subjective health status and medication usage were not included in multivariable models since they were considered to reflect consequences of having one or more overlapping conditions, and might therefore mask statistical associations attributable to the conditions themselves.

A second strategy of multivariable analysis used random forest modeling 23 to analyze potential contributions of all variables, not merely the reduced set of latent constructs. This novel method of data mining was used to achieve two goals: a) to identify the most important risk factors for first-onset TMD; and b) to generate plots depicting adjusted association between each variable and TMD incidence, with adjustment for the effects of other variables and with latitude in generating the plots that permitted departure from a straight-line association. The model produced importance scores, one for each variable, 
representing the decrease in the predictive accuracy of the model when the variable is measured incorrectly. The most important variable was assigned a score of 100, and all other importance scores have lower values that could range to a negative value if the variable worsened prediction. The random forest model was used also to compute the expected rate of first-onset TMD that would be observed at several values of the variable after averaging over the values of all other variables in the model. Partial dependence plots were then generated and LOESS smoothing was used to help visualize the association. ${ }^{26}$

The two strategies were selected in favor of other approaches for multivariable analysis for several reasons. The first strategy is a conventional approach that adjusts for potential confounding effects of variables identified a priori, based on conceptual relevance and univariate association with TMD incidence. However, it does not take advantage of information about the excluded variables. Thus, a random forest model was used to evaluate contributions of all variables. Random forests have several other advantages compared to conventional linear regression models. Specifically, random forests can impute for missing data and handle large numbers of correlated predictor variables without decreasing the accuracy of the model. ${ }^{21}$

\section{Sample Size Considerations}

OPPERA was designed with a target sample size of 3,200 enrolled study participants expected to yield 196 cases of first-onset TMD during a three-year follow-up period, assuming 30\% loss to follow-up. These targets were based on incidence and cohort retention rates observed in a previous study conducted at the N.C. study site, ${ }^{13}$ and were sufficient to provide statistical power of $80 \%$ to detect risk ratios of at least 1.8 for risk predictors with as few as $15 \%$ of in the high risk category, consistent with the magnitude of effect seen for genetic predictors seen in the previous N.C. study.

\section{RESULTS}

In 7,368 person-years of follow-up, 260 people developed first-onset TMD yielding an average annual incidence of 3.5\%.Two thirds (70.4\%) of the 260 incident cases reported having experienced TMD symptoms for one or two months in the three month period prior to the examination at which TMD-onset was determined, and 65\% said that their symptoms occurred in recurrent bouts.

Virtually all pain disorders assessed at enrollment were associated with greater TMD incidence (Table 1) and the relationship showed a dose-response pattern. For example, a simple count of the number of low back pain episodes in the year prior to enrollment revealed a dose-response relationship of increasing incidence of first-onset TMD with higher number of episodes, peaking at 5-10 episodes (HR=2.01 [95\% CL: 1.35, 3.00]). Among the 341 people with low back pain at enrollment, the incidence of first-onset TMD (site adjusted rate $=5.52 \%$ per annum) was twice the incidence seen in 2,379 people without low back pain $(2.92 \%$ per annum). The corresponding site-adjusted hazard ratio (HR=2.02 [95\% CL: $1.49,2.72]$ was only slightly attenuated after adjustment for demographic characteristics $(\mathrm{HR}=1.91$, [95\% CL: 1.42, 2.58]) and back pain remained a significant predictor of TMD after further adjustment for loss to follow-up (HR=1.89, 95\% [CL: 1.38, 2.57]).

Stronger effects were observed with irritable bowel syndrome (Table 1). Incidence of firstonset TMD was three times as high in people with IBS on enrolment as in people without IBS. Again the effect diminished on adjustment for demographic characteristics $(\mathrm{HR}=2.84$ [95\% CL: 1.75, 4.62]) and for loss to follow-up (HR=2.27 [95\% CL: 1.35, 3.79]; P=0.002) but IBS symptoms remained a significant predictor. As the count of IBS symptoms increased, so did incidence of first-onset TMD, such that people with six or more symptoms 
had 2.35 times greater rate of developing TMD than people with no IBS symptoms, in the fully adjusted model.

Incidence of first-onset TMD was three times higher in the 84 people with genital pain symptoms on enrolment than in 2,632 people without genital pain symptoms and incidence remained elevated in the fully adjusted model (HR=2.31 [95\% CL: 1.41, 3.77]).

When headache types were examined separately, clear differences emerged with tensiontype headache most strongly associated with TMD incidence (Table 1). Among the 206 people with tension-type headache at enrollment, TMD incidence was $69 \%$ greater than people who did not have tension-type headache (HR=1.69 [95\% CL: 1.12, 2.53]). Although TMD incidence was positively associated with migraine at enrollment, the hazard ratio attenuated to statistical non-significance after adjustment for demographic characteristics (HR=1.28 [95\% CL: 0.99, 1.64]. Meanwhile, probable tension-type headache at enrollment was not associated with TMD incidence. In a simple count of number of self-reported types of headaches, first-onset TMD incidence increased in monotonic fashion in accordance with the counts. Similarly, incidence of first-onset TMD increased with increasing intensity of headache.

Among other clinical conditions and characteristics reported at enrollment (Table 2) those significantly associated with increased incidence of TMD were a higher number of comorbid conditions, a history of neural conditions, a history of respiratory conditions, usage of three or more medications, current and former cigarette smoking, and a poorer than "excellent" self-rating of general health status. Strongest effects were observed for two or more comorbidities and poor/fair self-rated health. None of the potential putative risk factors emerged as being protective against first-onset TMD. Equally noteworthy were the conditions found not associated with TMD incidence: endocrine, cardiovascular or hematologic, sleep apnea and lifetime hospitalization for surgery or serious illness. However the low frequencies of endocrine conditions $(n=78)$ and sleep apnea $(n=47$ for) diminished the study's power to detect a true association if one exists.

The standardized hazard ratios for continuous variables (Table 3) are interpreted as the change in incidence of first-onset TMD associated with a one standard deviation change in the risk factor. For example, for each one standard deviation increase in PSQI score, denoting poorer sleep quality, incidence of first-onset TMD increased by 32\% (adjusted SHR $1.32,95 \%$ confidence limits (CL): 1.18, 1.47). A one standard deviation increase in finger length ratio (RD2:RD4) was associated with a 15\% increased incidence of developing first-onset TMD (adjusted SHR 1.15, 95\% CL: 1.01, 1.30), implying greater risk for people with lower testosterone and higher estrogen exposure in utero. In additional analyses that stratified by gender, the effect of 2D:4D ratio was stronger in females (HR 1.30, 95\% CL: $1.11,1.52$ ) than males (HR 1.07, 95\% CL: 0.86, 1.32), although the effect modification of gender was not significant $(\mathrm{P}=0.15)$ [results not tabulated]. Body mass index was a putative risk factor for first-onset TMD in analysis that adjusted for study site and demographic characteristics (Table 3), but its effect was attenuated to statistical non-significance after imputation for loss to follow-up. Higher summary scores on the SF12v2 physical and mental component reflect better health and functioning. Consistent with this coding, higher scores on both components were protective against development of first-onset TMD, and the effect was especially strong for the mental component (adjusted SHR 0.74 [95\% CL: 0.66, 0.82]).

The successive multivariable models (Table 4) demonstrated generally little confounding among overlapping conditions in their association with TMD incidence. For example, all five pain disorders were independent putative risk factors for first-onset TMD in model 2 with hazard ratios ranging from 1.80 for low back pain to 1.92 for both irritable bowel 
syndrome and genital symptoms. In model 3 that also adjusted for other health status conditions, sleep disturbances, current and former smoking and a high 2D:4D finger length ratio-denoting low testosterone exposure in utero-were independent risk factors for firstonset TMD. Also in this model 3, black race, low back pain and genital symptoms, but not tension-type headache or irritable bowel syndrome remained significant predictors. There was, however, evidence that other demographic associations were attenuated by effects of non-painful health conditions. For example, in model 1 risk of first-onset TMD increased by $20 \%$ for each 10 years of age and the effect was attenuated only by $3 \%$ with the inclusion in model 2 of the pain disorders. However, in model 3, in the presence of other clinical conditions, the effect of age reduced by a more marked increment of $10 \%$ per decade.

In the random forest model that predicted incidence using all health status variables reported in Tables 1-3 together with study site and demographics, incidence of TMD increased sharply as the number of comorbid conditions increased from 0 to 5, and thereafter flattened out. (Figure 1A) However, there was a threshold effect of headaches: TMD incidence among people with up to two headaches, but above that threshold, there was a sharp increase in incidence (Fig 1B). People who never smoked had markedly lower incidence of TMD compared to either current- or former-smokers (Figure 1C). While the model predicted a significantly higher rate of TMD in former smokers compared to current smokers, the absolute difference of the average-predicted rates was approximately 0.002 cases per annum (equivalent to a net difference of $0.2 \%$ per annum).

\section{DISCUSSION}

\section{Main Findings}

In this first large-scale prospective evaluation of the relationship between general health status and development of first-onset TMD, people with a history of lower back pain at enrollment had a 50\% greater incidence of TMD than people with no such history, after adjustment for other overlapping conditions. Similarly, a history of genital pain symptoms was associated with $75 \%$ greater incidence of developing TMD. Tension-type headache and irritable bowel syndrome predicted first-onset TMD after adjustment for demographic characteristics and pain disorders; however their effects were rendered statistically nonsignificant in the presence of other overlapping conditions. Some non-painful conditions at enrollment were independently associated with increased TMD incidence, including poor sleep quality.

This finding in a prospective cohort study is noteworthy by demonstrating that poor sleep is not solely a consequence of TMD pain. Another novel finding was that higher D2:D4 digit ratio, a marker of greater estrogen relative to testosterone exposure in utero, was associated with greater incidence of first-onset TMD. This lifelong signature of prenatal exposure poses the intriguing possibility of organizational hormonal contributions to TMD. Finally, this study provides the first evidence that current and former cigarette smoking increased incidence of first-onset TMD.

\section{Comparison with Previous Studies}

These results confirm and extend previous cross-sectional research of overlapping pain disorders. Consistent with the OPPERA baseline case-control study of chronic TMD, pain disorders in the present study were strongly associated with first-onset TMD. Also concordant with the OPPERA case control study, the present study found that cigarette smoking was strongly predictive of TMD. Although the OPPERA case control study found that TMD was associated with higher 2D:4D finger length ratios, the effect was nonsignificant in the fully adjusted model. ${ }^{35}$ 
An association between sleep disturbance and chronic pain is well established. Several longitudinal studies have found that persistent pain, including chronic orofacial pain, predicted onset of sleep disturbance. $8,36,38$ The relationship appears reciprocal. A Finnish cohort study ${ }^{24}$ followed industry workers for 28 years and examined two types of selfreported sleep disturbances as risk factors for incident hospitalization due to painful back disorders. Compared to workers with no sleep disturbances, those with one type of sleep disturbance had twice the risk of back disorder (HR 2.1; CL: 1.0, 4.6) and those with two types of sleep disturbance had three times the risk (HR 2.9; CL: 1.2, 7). ${ }^{24}$ Another study examined sleep duration in one night and subsequent pain reports the following day in a representative subset of the national Midlife in the United States Survey. ${ }^{15}$ Sleep duration of $\leq 3$ hours relative to 6-9 hours was associated with an $81 \%$ increase in pain frequency. ${ }^{3}$

The finding that a history of smoking increased incidence of first-onset TMD is consistent with other prospective cohort evidence of other pain conditions. Among a cohort of high school adolescents in Montreal, Canada, smoking was predictive of low back pain incidence occurring at least once a week within the past 6 months and the effect was dose responsive.$^{18}$ Similar dose response findings were reported in the Northern Finland 1986 Birth Cohort, although the effect was inconsistent in males. ${ }^{29}$ Among 4,472 18-30 year-olds who were followed for seven to 15 years in the CARDIA study, smokers had a 35\% higher risk than non-smokers of developing migraine headache (RR 1.35 [95\% CL: 1.08, 1.68]). ${ }^{22}$ In a four-year prospective cohort study in Norway of adults aged up to 32 years, those who were smokers at enrollment had greater risk than non-smokers of developing musculoskeletal pain in the neck, shoulder, upper back and lower back regions, and reported greater pain intensity than non-smokers. ${ }^{16}$

The 2D:4D digit ratio is informative of intra uterine exposure to sex hormones during the period of digit cartilage development in the fourteenth week of fetal life. ${ }^{48}$ Specifically, compared with females, males are exposed to higher fetal levels of testosterone relative to estrogen resulting in lower D2:D4 ratio. Evidence is growing that digit ratio predicts health outcomes, although the direction of the effect varies and the effect is sometimes seen in one hand only. In the left hand, higher 2D:4D ratio was associated with greater breast cancer risk, ${ }^{32}$ but with reduced odds of amyotrophic lateral sclerosis, a neurodegenerative disease in both men and women. ${ }^{46}$ Brabin et. al. ${ }^{6}$ showed that among female adolescents, high 2D: $4 \mathrm{D}$ ratio was associated with greater likelihood of persistent infection with human papilloma virus (HPV) and with greater occurrence of cervical intraepithelial neoplasia. The authors interpreted these findings to suggest that lower fetal androgen exposure may predispose women to develop persistent HPV with subsequent increased risk of cervical intraepithelial neoplasia. ${ }^{6}$

Another noteworthy finding was that non-painful health conditions to a large degree attenuated for the association between greater age and increased TMD incidence. In the OPPERA case-control study, greater age within this 18-44 year range was also associated with increased odds of chronic TMD, a result that was consistent with findings from the U.S. population, ${ }^{43}$ and which refutes views that TMD occurs most frequently in early adulthood. The current finding from multivariable modelling helps explain the age association, by showing that the association is mostly attributable to non-painful health conditions that increase with age. One implication is that TMD risk might be reduced if general health disorders could be prevented or managed early in adulthood.

\section{Strengths and Limitations}

The multi-site OPPERA project is a continuing prospective cohort study purposefully designed to investigate the etiology of first-onset TMD and variation in its genetic, biological and psychosocial determinants. It applied a rigorous methodology by using the 
same protocol and conditions to measure outcome and exposures in all subjects. This feature, along with the large size of the study, strengthens the validity of estimates. Findings should not be extrapolated beyond the study population or the condition studied: this was a generally healthy cohort of 18-44 year-olds and most participants who developed first-onset TMD had experienced symptoms for only one or two months. There are several limitations to the interpretation of findings that merit discussion. Despite demonstrating that a history of pain conditions, sleep disturbance, digit ratio and smoking preceded incident first-onset TMD, no finding is sufficient to infer a causal relationship. However, the new information does permit us to speculate about plausible mechanisms through which these predictors may operate. Although the Pittsburgh Sleep Quality Index is a psychometrically sound measure of sleep quality and disturbance, the subjective assessment falls short of evaluating specific sleep problems such as sleep disordered breathing for which measures such as hypoxemia and arousal may underlie this relationship. We believe this is an important area for future research using overnight sleep monitoring. This investigation would clarify whether hypoxemia plays a role in systemic inflammation in TMD pathophysiology. Some of the health status variables examined in this study were obtained by self-report of lifetime history. While this may introduce recall bias, there is no reason to believe that recall precision differs between with and without first-onset TMD. Hence any misclassification would bias findings toward the null hypothesis. Loss to follow up is an important source of bias when loss occurs more commonly in specific subgroups. The implications of this bias and our strategies for handling bias are discussed elsewhere (see Bair et. al.).

In conclusion, pre-existing pain conditions, sleep disturbance, cigarette smoking and high 2D:4D digit ratio were independent risk factors for first-onset TMD. This knowledge presents opportunities to explore aspects of general health and overlapping pain conditions for future therapeutic approaches to prevent TMD.

\section{Supplementary Material}

Refer to Web version on PubMed Central for supplementary material.

\section{Acknowledgments}

We are grateful to the study participants for their continuing contributions of time and effort in the OPPERA study.

\section{References}

1. Aaron LA, Buchwald D. Chronic diffuse musculoskeletal pain, fibromyalgia and co-morbid unexplained clinical conditions. Best Pract Res Clin Rheumatol. 2003; 17:563-74. [PubMed: 12849712]

2. Aaron LA, Burke MM, Buchwald D. Overlapping conditions among patients with chronic fatigue syndrome, fibromyalgia, and temporomandibular disorder. Arch Intern Med. 2000; 160:221-7. [PubMed: 10647761]

3. Anderson RJ, McCrae CS, Staud R, Berry RB, Robinson ME. Predictors of clinical pain in fibromyalgia: examining the role of sleep. J Pain. 2012; 13:350-8. [PubMed: 22381437]

4. Bair E, Brownstein N, Ohrbach RO, Greenspan JD, Dubner R, Fillingim RB, Diatchenko L, Smith S, Maixner W, Gonzalez Y, Gordon S, Lim P-F, Ribeiro-Dasilva M, Dampier D, Knott C, Slade GD. Study design, methods, sample characteristics and loss-to-follow-up: the OPPERA prospective cohort study. The journal of pain : official journal of the American Pain Society. in press (in this volume).

5. Berger MB, Damico NJ, Menees SB, Fenner DE, Haefner HK. Rates of Self-Reported Urinary, Gastrointestinal, and Pain Comorbidities in Women With Vulvar Lichen Sclerosus. J Low Genit Tract Dis. 2012 
6. Brabin L, Roberts SA, Farzaneh F, Fairbrother E, Kitchener HC. The second to fourth digit ratio (2D:4D) in women with and without human papillomavirus and cervical dysplasia. Am J Hum Biol. 2008; 20:337-41. [PubMed: 18203126]

7. Buysse DJ, Reynolds CF 3rd, Monk TH, Berman SR, Kupfer DJ. The Pittsburgh Sleep Quality Index: a new instrument for psychiatric practice and research. Psychiatry Res. 1989; 28:193-213. [PubMed: 2748771]

8. Cho CH, Jung SW, Park JY, Song KS, Yu KI. Is shoulder pain for three months or longer correlated with depression, anxiety, and sleep disturbance? J Shoulder Elbow Surg. 2012

9. Ciancaglini R, Radaelli G. The relationship between headache and symptoms of temporomandibular disorder in the general population. J Dent. 2001; 29:93-8. [PubMed: 11239582]

10. Dadabhoy D, Clauw DJ. Therapy Insight: fibromyalgia--a different type of pain needing a different type of treatment. Nat Clin Pract Rheumatol. 2006; 2:364-72. [PubMed: 16932722]

11. de Leeuw R, Studts JL, Carlson CR. Fatigue and fatigue-related symptoms in an orofacial pain population. Oral Surg Oral Med Oral Pathol Oral Radiol Endod. 2005; 99:168-74. [PubMed: 15660087]

12. Diatchenko L, Nackley AG, Slade GD, Fillingim RB, Maixner W. Idiopathic pain disorders-pathways of vulnerability. Pain. 2006; 123:226-30. [PubMed: 16777329]

13. Diatchenko L, Slade GD, Nackley AG, Bhalang K, Sigurdsson A, Belfer I, Goldman D, Xu K, Shabalina SA, Shagin D, Max MB, Makarov SS, Maixner W. Genetic basis for individual variations in pain perception and the development of a chronic pain condition. Hum Mol Genet. 2005; 14:135-43. [PubMed: 15537663]

14. Dworkin SF, LeResche L. Research diagnostic criteria for temporomandibular disorders: review, criteria, examinations and specifications, critique. J Craniomandib Disord. 1992; 6:301-55. [PubMed: 1298767]

15. Edwards RR, Almeida DM, Klick B, Haythornthwaite JA, Smith MT. Duration of sleep contributes to next-day pain report in the general population. Pain. 2008; 137:202-7. [PubMed: 18434020]

16. Eriksen W, Natvig B, Rutle O, Bruusgaard D. Smoking and the functional status of young adults. Scand J Prim Health Care. 1999; 17:174-9. [PubMed: 10555248]

17. Fantoni F, Salvetti G, Manfredini D, Bosco M. Current concepts on the functional somatic syndromes and temporomandibular disorders. Stomatologija. 2007; 9:3-9. [PubMed: 17449972]

18. Feldman DE, Rossignol M, Shrier I, Abenhaim L. Smoking. A risk factor for development of low back pain in adolescents. Spine (Phila Pa 1976). 1999; 24:2492-6. [PubMed: 10626312]

19. Glaros AG, Urban D, Locke J. Headache and temporomandibular disorders: evidence for diagnostic and behavioural overlap. Cephalalgia. 2007; 27:542-9. [PubMed: 17441972]

20. Goncalves DA, Camparis CM, Speciali JG, Franco AL, Castanharo SM, Bigal ME. Temporomandibular disorders are differentially associated with headache diagnoses: a controlled study. Clin J Pain. 2011; 27:611-5. [PubMed: 21368664]

21. Hastie, T.; Tibshirani, R.; Friedman, J. The elements of statistical learning: data mining, inference, and prediction. New York: Springer; 2009.

22. Hozawa A, Houston T, Steffes MW, Widome R, Williams OD, Iribarren C, Pletcher MJ, Daviglus ML, Carr JJ, Jacobs DR Jr. The association of cigarette smoking with self-reported disease before middle age: the Coronary Artery Risk Development in Young Adults (CARDIA) study. Prev Med. 2006; 42:193-9. [PubMed: 16480762]

23. Ishwaran H, Kogalur UB, Blackstone EH, Lauer MS. Random survival forests. The Annals of Applied Statistics. 2008; 2:841-60.

24. Kaila-Kangas L, Kivimaki M, Harma M, Riihimaki H, Luukkonen R, Kirjonen J, Leino-Arjas P. Sleep disturbances as predictors of hospitalization for back disorders-a 28-year follow-up of industrial employees. Spine (Phila Pa 1976). 2006; 31:51-6. [PubMed: 16395176]

25. Lim PF, Maixner W, Khan AA. Temporomandibular disorder and comorbid pain conditions. J Am Dent Assoc. 2011; 142:1365-7. [PubMed: 22130437]

26. Loader, C. Local regression and likelihood. New York: Springer; 1999. 
27. Maixner W, Diatchenko L, Dubner R, Fillingim RB, Greenspan JD, Knott C, Ohrbach R, Weir B, Slade GD. Orofacial pain prospective evaluation and risk assessment study--the OPPERA study. J Pain. 2011; 12:T4-11. e1-2. [PubMed: 22074751]

28. Manning JT, Callow M, Bundred PE. Finger and toe ratios in humans and mice: implications for the aetiology of diseases influenced by HOX genes. Med Hypotheses. 2003; 60:340-3. [PubMed: 12581609]

29. Mikkonen P, Leino-Arjas P, Remes J, Zitting P, Taimela S, Karppinen J. Is smoking a risk factor for low back pain in adolescents? A prospective cohort study. Spine (Phila Pa 1976). 2008; 33:527-32. [PubMed: 18317198]

30. Mitchell MD, Mannino DM, Steinke DT, Kryscio RJ, Bush HM, Crofford LJ. Association of smoking and chronic pain syndromes in Kentucky women. J Pain. 2011; 12:892-9. [PubMed: 21816352]

31. Mohl ND, Ohrbach R. Clinical decision making for temporomandibular disorders. J Dent Educ. 1992; 56:823-33. [PubMed: 1487585]

32. Muller DC, Baglietto L, Manning JT, McLean C, Hopper JL, English DR, Giles GG, Severi G. Second to fourth digit ratio (2D : 4D), breast cancer risk factors, and breast cancer risk: a prospective cohort study. Br J Cancer. 2012

33. Mundt T, Mack F, Schwahn C, Bernhardt O, Kocher T, Biffar R. Association between sociodemographic, behavioral, and medical conditions and signs of temporomandibular disorders across gender: results of the study of health in Pomerania (SHIP-0). Int J Prosthodont. 2008; 21:141-8. [PubMed: 18546769]

34. Nickel JC, Tripp DA, Pontari M, Moldwin R, Mayer R, Carr LK, Doggweiler R, Yang CC, Mishra $\mathrm{N}$, Nordling J. Interstitial cystitis/painful bladder syndrome and associated medical conditions with an emphasis on irritable bowel syndrome, fibromyalgia and chronic fatigue syndrome. J Urol. 2010; 184:1358-63. [PubMed: 20719340]

35. Ohrbach R, Fillingim RB, Mulkey F, Gonzalez Y, Gordon S, Gremillion H, Lim PF, RibeiroDasilva M, Greenspan JD, Knott C, Maixner W, Slade G. Clinical findings and pain symptoms as potential risk factors for chronic TMD: descriptive data and empirically identified domains from the OPPERA case-control study. J Pain. 2011; 12:T27-45. [PubMed: 22074750]

36. Palermo TM, Wilson AC, Lewandowski AS, Toliver-Sokol M, Murray CB. Behavioral and psychosocial factors associated with insomnia in adolescents with chronic pain. Pain. 2011; 152:89-94. [PubMed: 21030151]

37. Peters KM, Killinger KA, Mounayer MH, Boura JA. Are ulcerative and nonulcerative interstitial cystitis/painful bladder syndrome 2 distinct diseases? A study of coexisting conditions. Urology. 2011; 78:301-8. [PubMed: 21703668]

38. Riley JL 3rd, Benson MB, Gremillion HA, Myers CD, Robinson ME, Smith CL Jr, Waxenberg LB. Sleep disturbance in orofacial pain patients: pain-related or emotional distress? Cranio. 2001; 19:106-13. [PubMed: 11842861]

39. Rodriguez MA, Afari N, Buchwald DS. Evidence for overlap between urological and nonurological unexplained clinical conditions. J Urol. 2009; 182:2123-31. [PubMed: 19758633]

40. Sancisi E, Cevoli S, Vignatelli L, Nicodemo M, Pierangeli G, Zanigni S, Grimaldi D, Cortelli P, Montagna P. Increased prevalence of sleep disorders in chronic headache: a case-control study. Headache. 2010; 50:1464-72. [PubMed: 20572880]

41. Sanders AE, Maixner W, Nackley AG, Diatchenko L, By K, Miller VE, Slade GD. Excess risk of temporomandibular disorder associated with cigarette smoking in young adults. J Pain. 2012; 13:21-31. [PubMed: 22036516]

42. Scher AI, Stewart WF, Lipton RB. The comorbidity of headache with other pain syndromes. Headache. 2006; 46:1416-23. [PubMed: 17040338]

43. Slade GD, Bair E, By K, Mulkey F, Baraian C, Rothwell R, Reynolds M, Miller V, Gonzalez Y, Gordon S, Ribeiro-Dasilva M, Lim PF, Greenspan JD, Dubner R, Fillingim RB, Diatchenko L, Maixner W, Dampier D, Knott C, Ohrbach R. Study methods, recruitment, sociodemographic findings, and demographic representativeness in the OPPERA study. J Pain. 2011; 12:T12-26. [PubMed: 22074749] 
44. Smith MT, Wickwire EM, Grace EG, Edwards RR, Buenaver LF, Peterson S, Klick B, Haythornthwaite JA. Sleep disorders and their association with laboratory pain sensitivity in temporomandibular joint disorder. Sleep. 2009; 32:779-90. [PubMed: 19544755]

45. Stone AA, Broderick JE. Obesity and pain are associated in the United States. Obesity (Silver Spring). 2012; 20:1491-5. [PubMed: 22262163]

46. Vivekananda U, Manjalay ZR, Ganesalingam J, Simms J, Shaw CE, Leigh PN, Turner MR, AlChalabi A. Low index-to-ring finger length ratio in sporadic ALS supports prenatally defined motor neuronal vulnerability. J Neurol Neurosurg Psychiatry. 2011; 82:635-7. [PubMed: 21551173]

47. Wiesinger B, Malker H, Englund E, Wanman A. Does a dose-response relation exist between spinal pain and temporomandibular disorders? BMC Musculoskelet Disord. 2009; 10:28. [PubMed: 19254384]

48. Zheng Z, Cohn MJ. Developmental basis of sexually dimorphic digit ratios. Proc Natl Acad Sci U S A. 2011; 108:16289-94. [PubMed: 21896736] 


\section{Perspective}

This article examines health conditions that commonly overlap with TMD to determine which predict first-onset TMD. A history of lower back pain and genital pain conditions at baseline were important predictors. Novel findings were that disrupted sleep and conditions in utero may increase incidence of first-onset TMD. 


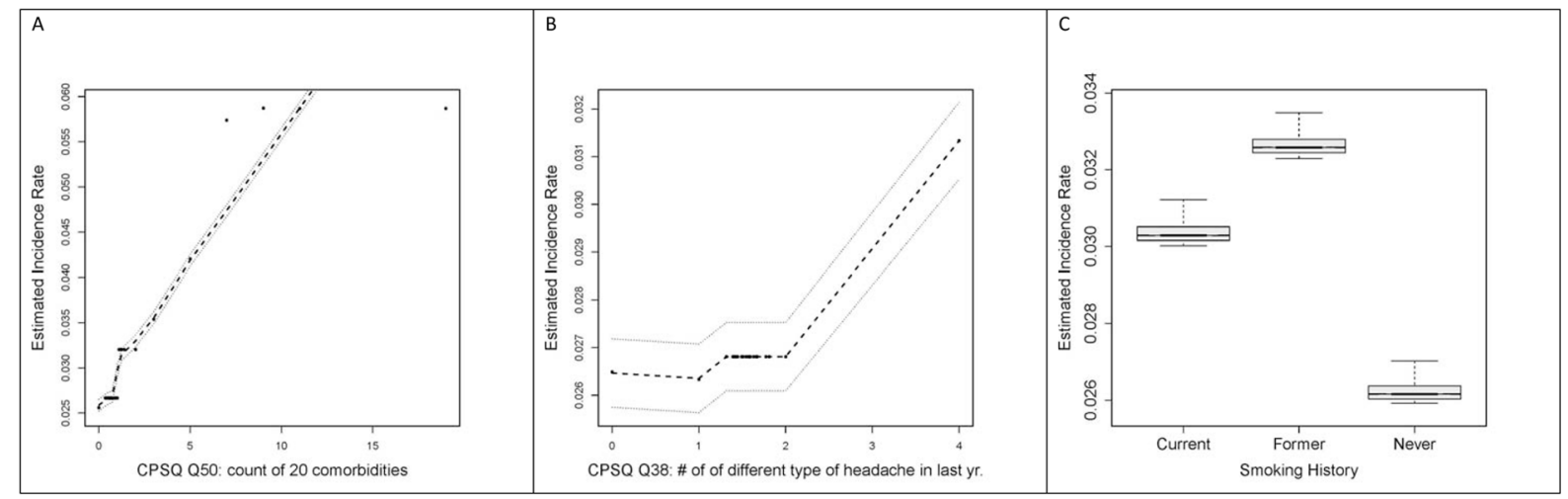

Figure 1.

Predicted TMD incidence rates from random forest models: OPPERA prospective cohort study, 2006-2011

TMD incidence rates, expressed as cases per 100-person-years, were generated from random forest models that predicted TMD onset using study site and sociodemographic variables reported in Tables 1-3. For continuous variables in A and B, Predicted values $(-)$ are plotted together with LOESS-smoothed estimates (- - ) and their 95\% confidence intervals (.....). For categories reported in $\mathrm{C}$, boxes represent interquartile range of predicted incidence rates, and horizontal lines within boxes represent the median (full width of box) and mean (half-width of box). Whiskers extend above each box to whichever is the lesser of the maximum value or 1.5 times the interquartile range above the third quartile. Whiskers extend below each box to whichever is the greater of the minimum value or 1.5 times the interquartile range below the third quartile. 


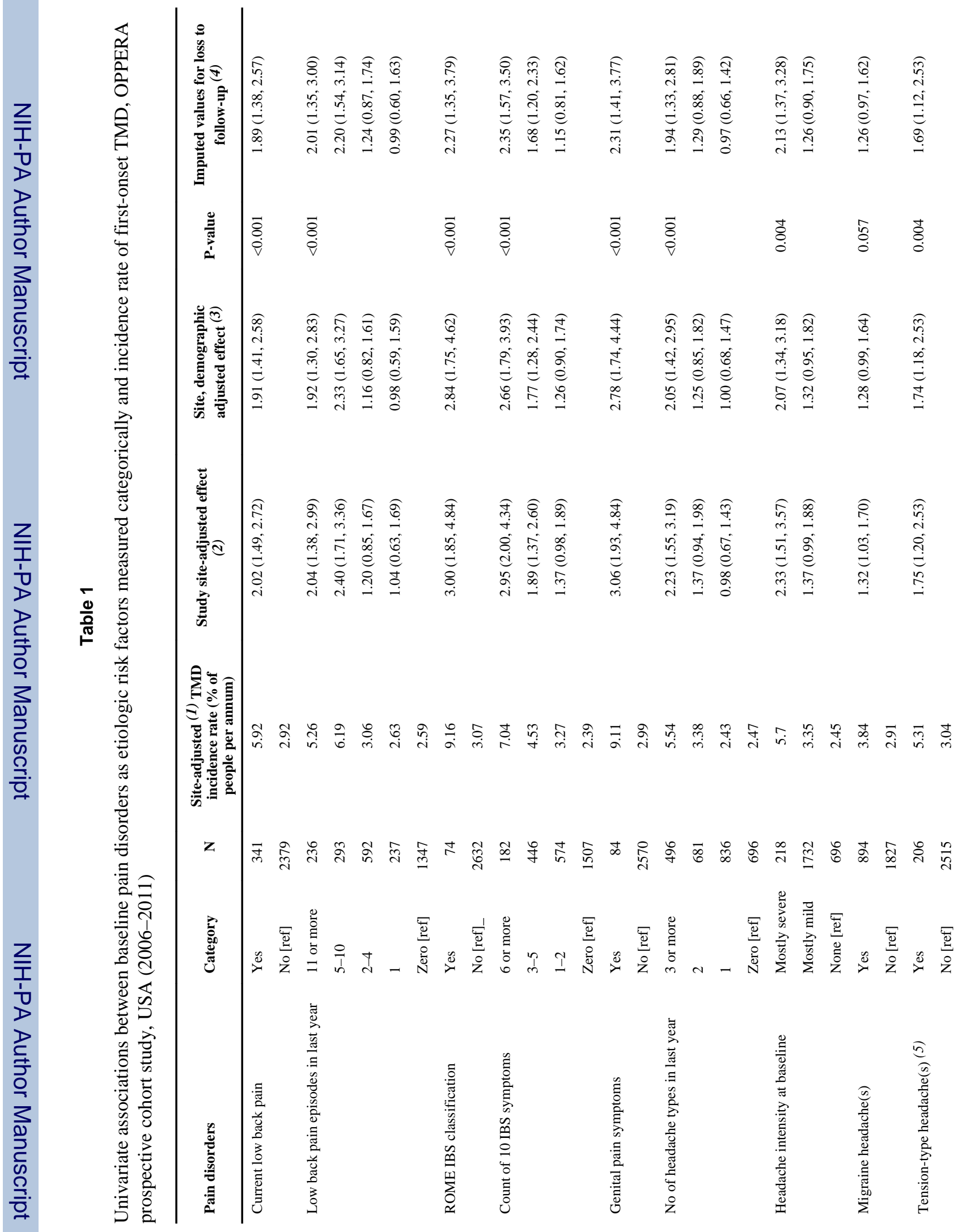


Sanders et al.

Page 18

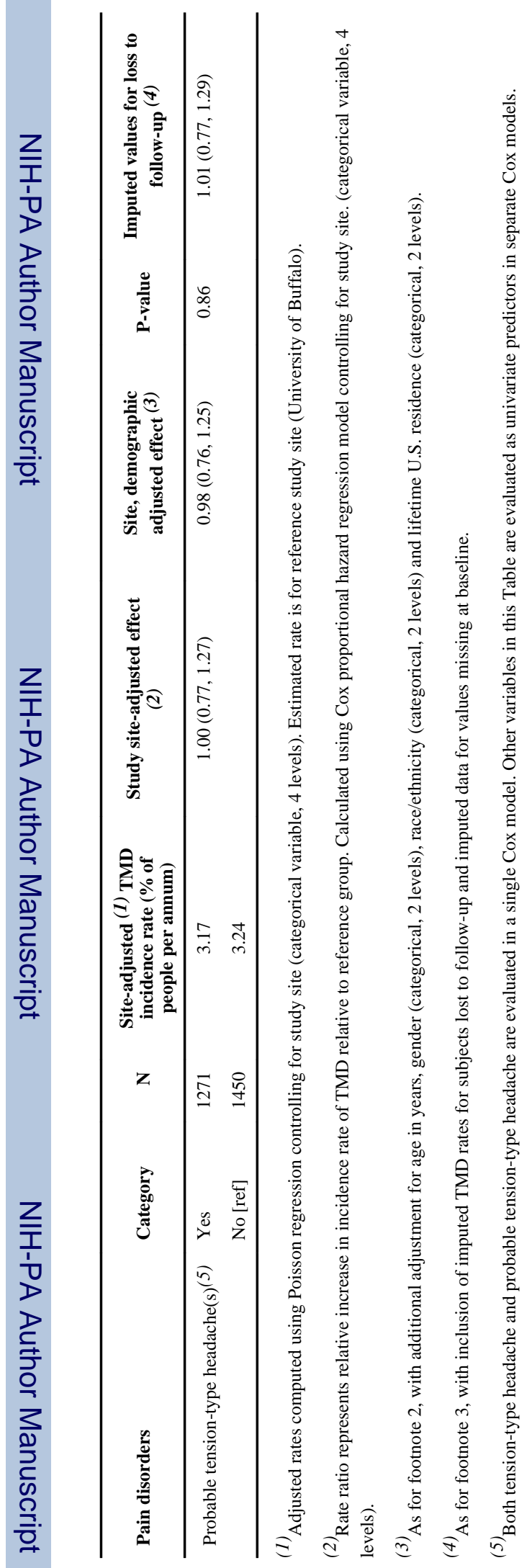

J Pain. Author manuscript; available in PMC 2014 December 01. 


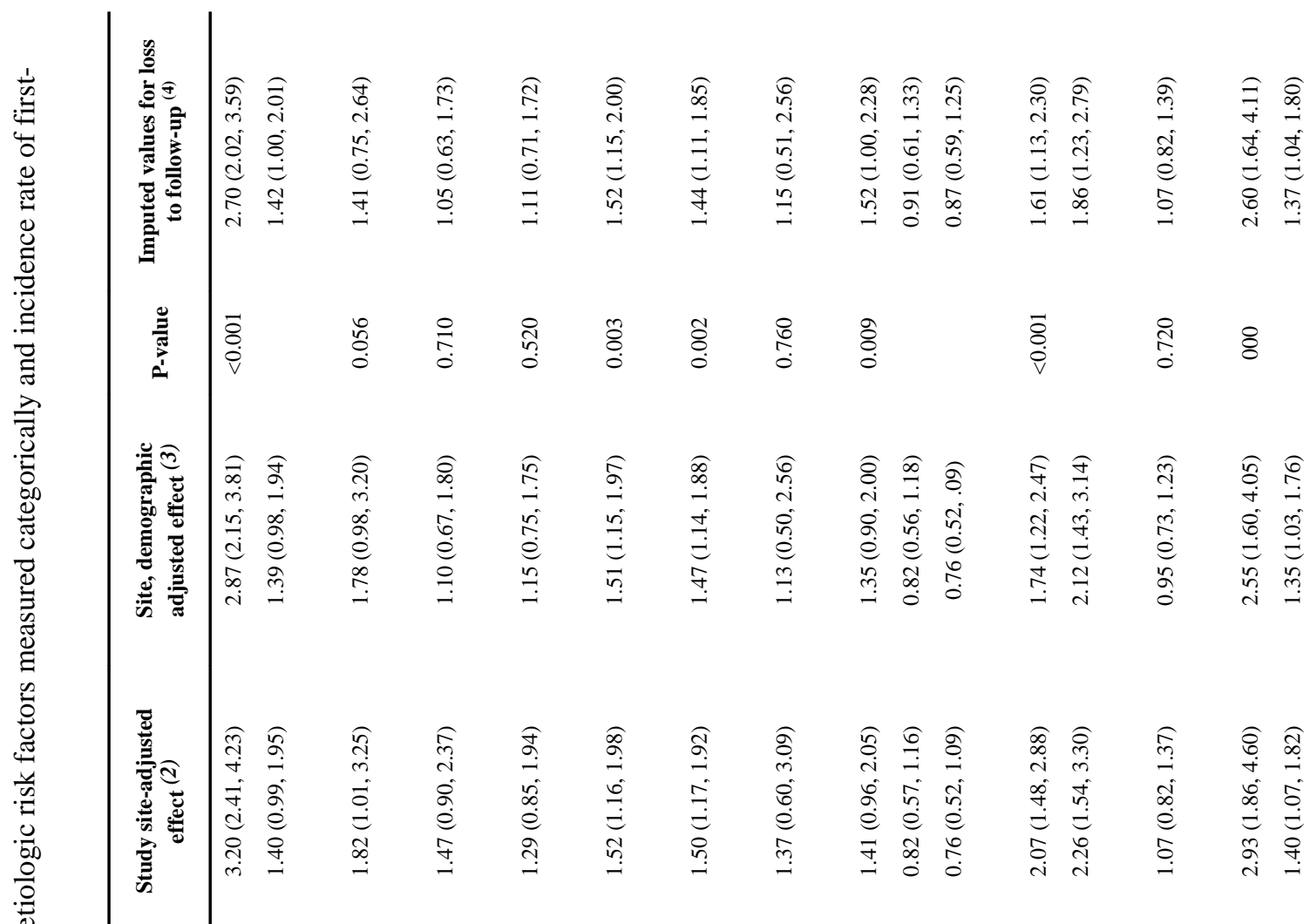

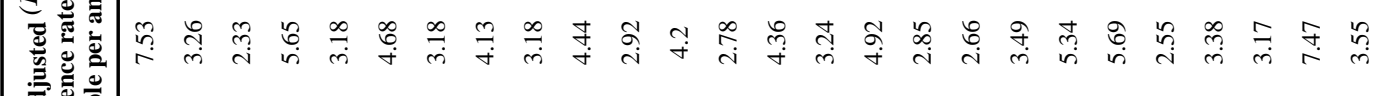

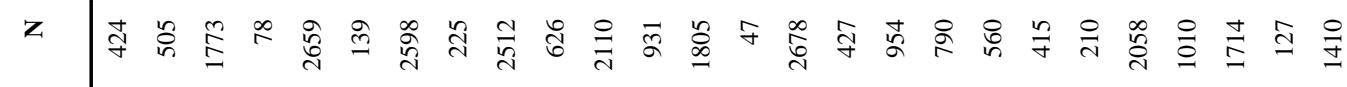

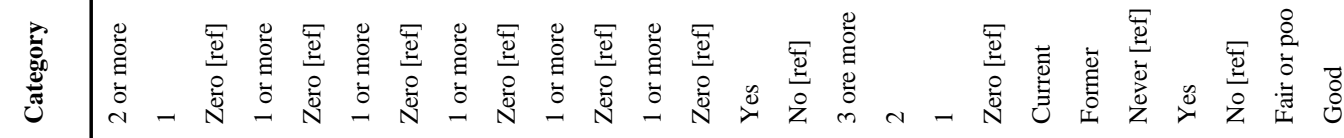
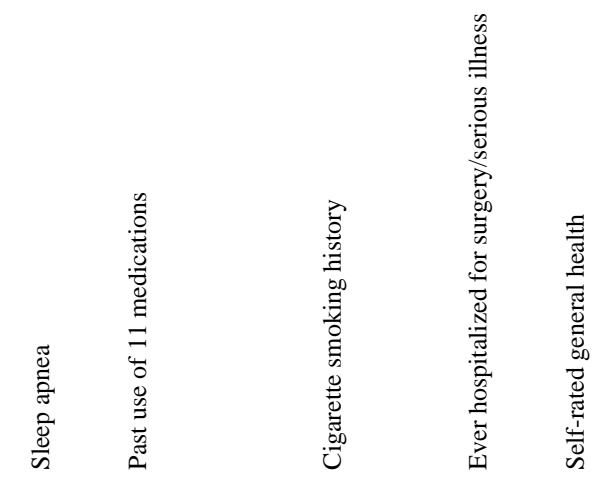


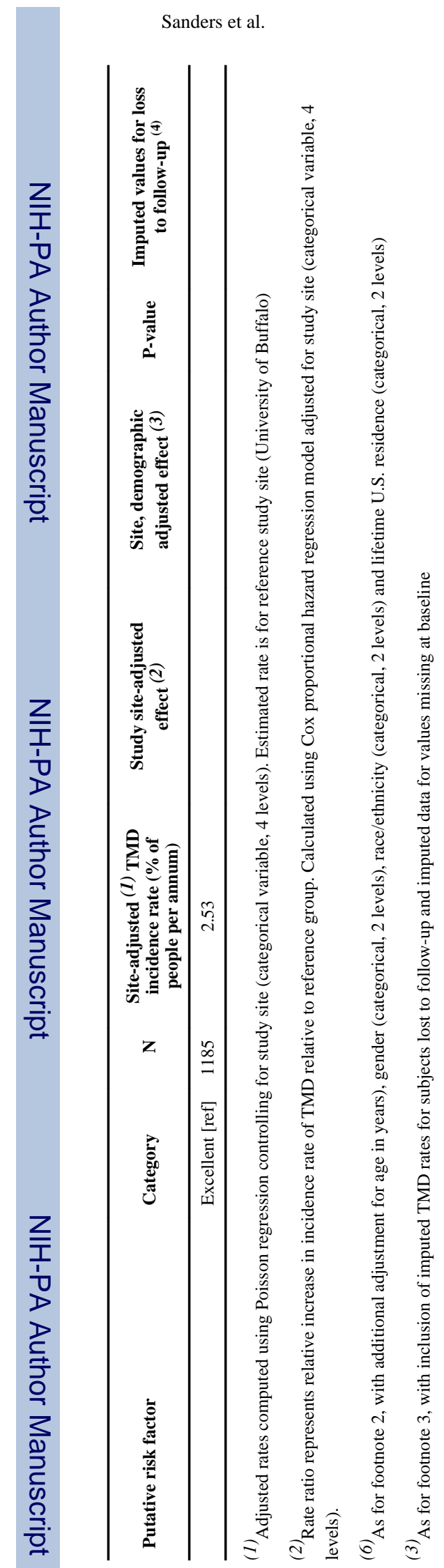

$J$ Pain. Author manuscript; available in PMC 2014 December 01. 


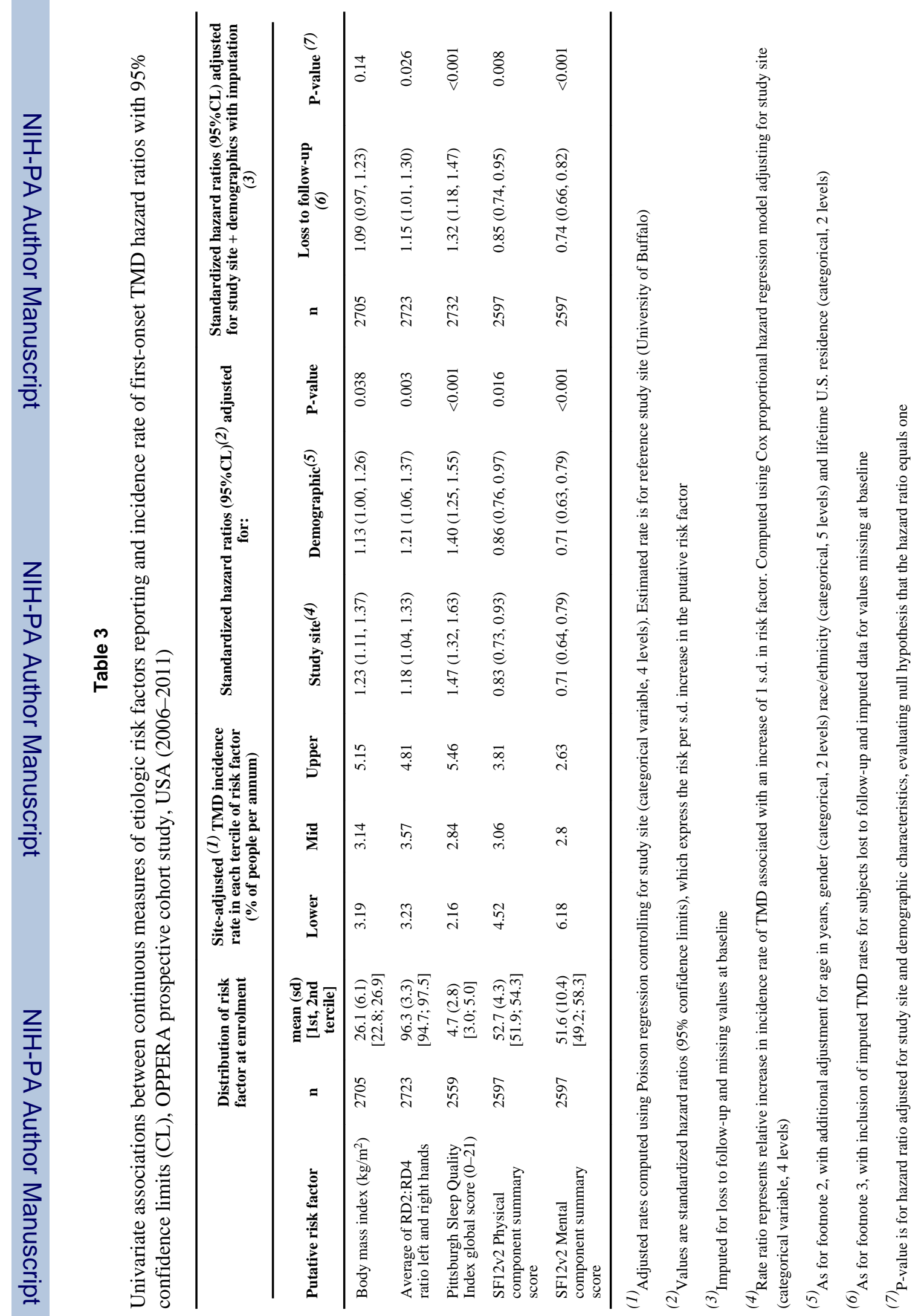

J Pain. Author manuscript; available in PMC 2014 December 01. 
Table 4

Multivariable-adjusted associations in development of first-onset TMD, OPPERA prospective cohort study, USA (2006-2011)

\begin{tabular}{|c|c|c|c|}
\hline & $\begin{array}{c}\text { Model } 1(1) \\
\text { Demographics }\end{array}$ & Model $2^{(1)}$ Pain disorders & $\begin{array}{l}\text { Model } 3 \text { (1) Other health } \\
\text { conditions }\end{array}$ \\
\hline & HR $(95 \% \mathrm{CL})$ & HR $(95 \% \mathrm{CL})$ & HR $(95 \%$ CL) \\
\hline Female gender $[\mathrm{ref}=\mathrm{male}]$ & $1.37(1.04,1.80)$ & $1.28(0.96,1.69)$ & $1.22(0.91,1.62)$ \\
\hline Age (in decades) & $1.20(1.01,1.43)$ & $1.17(0.98,1.39)$ & $1.07(0.88,1.28)$ \\
\hline Asian race $[$ ref=white $]$ & $0.66(0.31,1.40)$ & $0.67(0.32,1.43)$ & $0.71(0.33,1.50)$ \\
\hline Black race $[$ ref=white $]$ & $1.33(0.97,1.84)$ & $1.36(0.99,1.88)$ & $1.47(1.05,2.05)$ \\
\hline Hispanic ethnicity [ref=white] & $1.17(0.63,2.14)$ & $1.19(0.64,2.18)$ & $1.22(0.66,2.26)$ \\
\hline Other race $[$ ref=white $]$ & $0.96(0.45,2.07)$ & $1.04(0.48,2.25)$ & $1.08(0.49,2.34)$ \\
\hline Non-lifetime U.S. residence [ref=lifetime] & $0.46(0.27,0.78)$ & $0.47(0.28,0.79)$ & $0.51(0.30,0.86)$ \\
\hline Current low back pain $[\mathrm{ref}=\mathrm{no}]$ & & $1.80(1.30,2.48)$ & $1.50(1.08,2.10)$ \\
\hline ROME IBS classification $[\mathrm{ref}=\mathrm{no}]$ & & $1.92(1.12,3.30)$ & $1.62(0.94,2.81)$ \\
\hline Genital symptoms $[\mathrm{ref}=\mathrm{no}]$ & & $1.92(1.15,3.19)$ & $1.75(1.04,2.93)$ \\
\hline Genital symptoms not stated $[\mathrm{ref}=\mathrm{no}]$ & & $1.84(1.04,3.27)$ & $1.68(0.94,3.00)$ \\
\hline Tension-type headache $[\mathrm{ref}=\mathrm{no}]$ & & $1.57(1.04,2.35)$ & $1.46(0.97,2.20)$ \\
\hline Neurological conditions $[\mathrm{ref}=\mathrm{no}]$ & & & $1.25(0.93,1.68)$ \\
\hline Respiratory conditions $[\mathrm{ref}=\mathrm{no}]$ & & & $1.28(0.98,1.67)$ \\
\hline Pittsburgh Sleep Quality Index (z-score) & & & $1.18(1.05,1.33)$ \\
\hline $\mathrm{RD} 2: \mathrm{RD} 4$ ratio average both hands (z-score) & & & $1.15(1.00,1.31)$ \\
\hline Current smoker [ref=Never smoked] & & & $1.55(1.08,2.25)$ \\
\hline Former smoker [ref=Never smoked] & & & $1.84(1.22,2.78)$ \\
\hline Smoking status unstated [ref=Never smoked] & & & $1.39(0.63,3.04)$ \\
\hline
\end{tabular}

${ }^{(1)}$ All models are adjusted for study site 